\title{
Avaliação do risco cardiovascular e da severidade da doença renal em pacientes em
}

\section{hemodiálise}

Paloma Lopes Fonseca ${ }^{1}$, Lucyana Bertoso de Vasconcelos Freire ${ }^{2}$

${ }^{1}$ Enfermeira, Especialista em Nefrologia pelo Programa de Residência em Enfermagem em Nefrologia da ESCS/FEPECS/SES-DF, Brasília (DF), Brasil https://orcid.org/0000-00016537-9101.

${ }^{2}$ Enfermeira Nefrologista, Tutora do Programa de Residência em Enfermagem em Nefrologia da ESCS/FEPECS/SES, Mestre em Política, Práticas e Cuidado em Saúde e Enfermagem/UnB. Brasília (DF), Brasil.

Endereço para correspondências paloma.lop94@hotmail.com

\section{RESUMO}

Objetivo: analisar o risco cardiovascular (RCV) e a severidade da doença renal em pacientes em hemodiálise (HD). Métodos: estudo investigativo, transversal e quantitativo, com amostra de 54 pacientes em HD.Foram utilizados o Escore de Risco de Framingham (ERF) para calcular o RCV e o Índice de Severidade da Doença Renal (ISDR). Resultados:os dados apontaram para médio e alto RCV $(61,10 \%)$ segundo o ERF. Os fatores de risco mais prevalentes pelo ERF foram hipertensão arterial sistêmica, sedentarismo e consumo elevado de sódio. De acordo com o ISDR, foram identificados $81,48 \%$ dos pacientes com alto e médio risco de severidade da DRC. As variáveis mais evidentes no ISDR foram a diminuição da acuidade visual e acesso para a diálise. Houve associação estatística entre o ERF e o consumo elevado de sódio e entre o ERF e o ISDR. Conclusão: O médio e alto risco cardiovascular e médio e alto risco de severidade da doença renal foram prevalentes nos pacientes renais em HD do estudo. Deve-se priorizar o atendimento multidisciplinar, por meio do levantamento de estratégias para minimizar riscos e promover a adesão ao tratamento, a fim de melhorar a sobrevida e qualidade de vida dessa população.

Palavras-chave: Diálise Renal; Doenças Cardiovasculares; Medição de Risco; Insuficiência Renal; Complicações; Doença Crônica.

\section{ABSTRACT}

Objective: to analyze cardiovascular risk (CVR) and the severity of kidney disease in hemodialysis (HD) patients. Methods: an investigative, cross-sectional and quantitative study with a sample of 54 patients on HD. The Framingham Risk Score (ERF) was used to calculate the CVR and the Kidney Disease Severity Index (ISDR). Results: the data pointed to medium and high RCV $(61.10 \%)$ according to the ERF. The most prevalent risk factors for ERF were systemic arterial hypertension, physical inactivity and high sodium consumption. According to the ISDR, $81.48 \%$ of patients with high and medium risk of severity of CKD were identified. The most evident variables in the ISDR were the decrease in visual acuity and access to dialysis. There was a statistical association between ERF and high sodium consumption and between ERF and ISDR. Conclusion: Medium and high cardiovascular risk and medium and high risk of kidney disease severity were prevalent in HD renal patients in the study. Priority should be given to multidisciplinary care, by surveying strategies to minimize risks and promote adherence to treatment, in order to improve the survival and quality of life of this population.

Keywords: Renal dialysis; Cardiovascular diseases; Renal insufficiency Risk Measurement; Complications;Chronic Disease. 


\section{INTRODUÇÃO}

A Doença Renal Crônica (DRC), de acordo com a National Kidney Foundation, é determinada a partir do nível de função renal do paciente e dividida em 5 estágios funcionais. $\mathrm{O}$ estadiamento cursa desde lesão renal com função normal à falência renal ou Doença Renal Crônica Terminal (DRCT), na qual o paciente necessita de terapia renal substitutiva (TRS). ${ }^{1}$

A DRC, quando não tratada, pode evoluir para uma DRCT, quando a taxa de filtração glomerular (TFG) atinge valores inferiores a $15 \mathrm{~mL} / \mathrm{min} / 1,73 \mathrm{~m} 2$, estabelecendo-se falência funcional renal (FFR). A DRC representa um sério problema de saúde pública em todo o mundo, sendo considerada uma epidemia de crescimento alarmante. ${ }^{1}$

Segundo o censo de 2016 da Sociedade Brasileira de Nefrologia (SBN), estima-se que 122.825 pacientes sigam em tratamento dialítico no Brasil, sendo a hemodiálise (HD) o tratamento mais recorrente. Atualmente, cerca de 1,5 milhão de pessoas vivem com DRC no Brasil e cerca de 30 mil novos casos por ano são registrados de pessoas que atingem a fase mais crítica da doença necessitando de TRS. ${ }^{2}$ Segundo dados da SBN de 2016, o número de unidades de HD no Brasil vem crescendo, passando de 510 no ano de 2000 para 747 unidades em $2016 .^{2}$

A mortalidade por DRC é 10 (dez) a 20 (vinte) vezes maior que a da população geral. Trata-se de uma doença insidiosa, grave, com profundos impactos na qualidade, sobrevida e expectativa de vida do indivíduo. ${ }^{3}$

Na procura em melhorar a sobrevida do paciente dialítico e reduzir as altas taxas de mortalidade, estudiosos têm desenvolvido instrumentos que medem os riscos relacionados à doença e ao tratamento e também o grau de severidade ou impacto que a doença provoca ao longo dos anos. Essas ferramentas são utilizadas tanto na prática clínica quanto em pesquisas oferecendo a oportunidade de melhor comparar resultados em diferentes populações em pacientes em diálise e levantar estratégias eficazes de prevenção e controle de agravos relacionados à patologia.

Quando se trata da função regulatória e hemodinâmica em nosso organismo, os sistemas cardiovascular e renal formam um sistema integrado ${ }^{4}$. Doenças Cardiovasculares e DRC, quando associadas, acarretam a piora da função renal o que favorece ao aumento do risco de DCV com consequente progressão inexorável da doença renal ${ }^{5}$. Aproximadamente 
$50 \%$ dos óbitos em pacientes em estágio final da DRC são atribuídos à DCV ${ }^{1}$. Em um estudo realizado por Azevedo, no Centro Pró-Renal de Barbacena, no período de janeiro de 1999 a julho de 2004, foi verificado que as principais causas de morte em dialíticos eram: infecções $(23,9 \%)$ e neoplasias (13\%) e em destaque doenças cardiovasculares $(45 \%)^{6}$

Existem métodos para avaliação de risco de DCV. Eles permitem identificar indivíduos em risco cardiovascular e medir o grau de exposição ao agravo e avaliar a necessidade e a intensidade da intervenção terapêutica. O reconhecimento do risco permite incentivar os indivíduos a alterar comportamentos prejudiciais e, de acordo com o valor de risco obtido, definir a periodicidade da vigilância médica para estabelecer metas terapêuticas?

Uma das ferramentas mais empregadas para esse fim é o Escore de Risco de Framingham (ERF), que trata de uma ferramenta mundialmente reconhecida e utilizada para o cálculo e estimativa de DCV na prática clínica, além de estratificar o risco para DVC . Criada a partir do Framingham Heart Study, sua aplicação teve início em 1948, com o objetivo de estimar os fatores responsáveis pelas mortes por DCV a partir de dados como colesterol, diabetes, gênero, idade, pressão sanguínea e tabagismo ${ }^{8-10}$. O ERF é capaz de estimar o risco de desenvolvimento de DCV em um período de 10 (dez) anos, o que possibilita formar grupos específicos de pacientes que se beneficiarão da terapia medicamentosa como forma de intervenção primária na prevenção de $\mathrm{DVC}^{8}$.

Quanto ao grau de severidade de uma doença é relacionada ao nível de distúrbios fisiológicos de um paciente com determinada enfermidade. A avaliação da severidade de uma doença se faz necessário para comparar amostras de pacientes e investigar os fatores que determinam a mesma. Um instrumento validado para avaliar a severidade da doença renal é denominado Índice De Severidade Da Doença Renal (ISDR) proposto por Craven. Esse método foi desenvolvido na Universidade de Toronto para avaliar a influência dos aspectos de comorbidades na evolução dos pacientes renais crônicos ${ }^{11}$.

Em serviços de Diálise, é importante a utilização de protocolos para acompanhamento de risco cardiovascular e da severidade da doença renal. Considerando-se a relevância da temática, é necessário dispor de tais recursos para estimar o Risco Cardiovascular (RCV) e o grau de severidade ou impacto da doença crônica dos pacientes submetidos a terapia hemodialítica. 
Os residentes de Enfermagem em Nefrologia, desenvolvem atividades em serviços que atendem pacientes em terapia hemodialítica e na prática clínica observaram a prevalência de complicações e morbidades que se apresentavam associadas às comorbidades da doença renal e DCVs. Neste contexto, o pesquisador desenvolveu o presente estudo com o objetivo de analisar o risco cardiovascular e a severidade da doença renal dos pacientes submetidos à hemodiálise.

\section{MÉTODO}

Trata-se de um estudo descritivo, exploratório, transversal, com abordagem quantitativa. Realizado em uma Unidade de Hemodiálise de um Hospital da Secretaria de Estado de Saúde do Distrito Federal. Uma instituição de grande porte que atende pacientes renais crônicos em terapia hemodialítica. O setor conta com 04 (quatro) salas que realizam hemodiálise, divididas quanto às sorologias (hepatites B, HIV (Human Immunodeficiency Vírus), sorologia negativa e desconhecida), num total de 20 (vinte) máquinas de hemodíalise. A equipe multiprofissional é formada por médicos nefrologistas, enfermeiros especialistas em nefrologia, técnicos de enfermagem capacitados em HD, psicóloga e assistente social. O regime dialítico dos pacientes consiste em sessões de diálise de 04 (quatro) horas realizadas 03 (três) vezes por semana, nas quais são divididas em turnos manhã, tarde e noite, de segunda a sábado.

A população do estudo foi composta por pacientes renais crônicos em terapia hemodialítica. A amostra do estudo foi determinada de acordo com os critérios de inclusão e exclusão pré-estabelecidos. Foram incluídos na pesquisa pacientes renais crônicos, de ambos os gêneros, acima de 18 anos, em regime hemodialítico crônico e que concordaram participar do estudo assinando o Termo de Consentimento Livre e Esclarecido (TCLE). Foram excluídos pacientes em condições clínicas que impossibilitaram a participação, pacientes com exames incompletos, ilegíveis, ou não coletados, pacientes com prontuários ilegíveis e não encontrados.

No período da coleta de dados, realizado de maio a agosto de 2017, havia no cenário do estudo cerca de 88 pacientes em hemodiálise, a amostra foi constituída de 54 pacientes. Foram excluídos 34 pacientes, sendo eles, pacientes submetidos à diálise por condições agudas (13), sem exames ou exames incompletos (08), transferidos para outra unidade de 
diálise (05), sem condições clínicas (05), óbito (02) e aqueles que não aceitaram participar da pesquisa (01), resultando em uma amostra de 54 pacientes.

Os dados foram coletados pelos pesquisadores por meio de entrevista aos participantes durante as sessões de hemodiálise e por meio de análise documental (prontuários físicos e eletrônicos). Foi utilizado formulário estruturado elaborado pelos próprios pesquisadores contendo questões abertas e fechadas com o intuito de contemplar os objetivos do estudo. O formulário estruturado foi construído em VI partes. A parte I contemplou questões relativas ao perfil epidemiológico; a parte II , aos dados clínicos, laboratoriais e antropométricos; a parte III, às comorbidades e a parte IV contemplou questões relacionadas aos hábitos de vida do paciente e a parte V e VI continham os instrumentos validados para medição do risco cardiovascular e da severidade da doença renal descritos a seguir.

Para a avaliação do risco cardiovascular, foi utilizado o Escore de Risco de Framingham (ERF) utilizado para estimar o risco do desenvolvimento de doenças cardiovasculares em um período de 10 (dez anos), os parâmetros utilizados para o cálculo do Risco Cardiovascular são, idade, LDL (Low Density Lipoproteins), HDL (High Density Lipoprotein), pressão arterial sistólica (PAS) e diastólica (PAD) e ainda a presença ou não de Diabetes mellitus (DM) e tabagismo. As pontuações são diferentes de acordo o gênero do paciente. Na etapa 1, são calculados os pontos de acordo idade, LDL, HDL, PAS e PAD, se possui DM e se é tabagista. Na etapa 2, é realizada a soma dos pontos e por fim na etapa 3, é relacionado o total de pontos ao percentual do RCV e identificado a estratificação do risco em baixo (<10\%), médio (10 a 20\%) ou alto (>20\%).

Para avaliar a severidade da doença renal foi utilizado o Índice de Severidade da Doença Renal (ISDR). O instrumento avalia a influência dos aspectos de comorbidades na evolução dos pacientes renais crônicos. O ISDR é composto de dez categorias de doenças, que são comuns em urêmicos: Cardiovascular, cerebrovascular, doença vascular periférica, neuropatia periférica, doença óssea, doença respiratória, deficit visual, neuropatia autonômica e doença gastrointestinal, acesso e ocorrências dialíticas, Diabetes Mellitus (DM) e uma categoria aberta.

A soma total do escore resulta na classificação da severidade da doença ${ }^{10}$. Cada categoria possui 3 variações de severidade: leve moderado e severo, com graduação: 1-3 para 
alterações leves ou bioquímicas de doença; 4-6 para sinais físicos moderados a severo; 7-8 para uma alteração física debilitante ou prognóstico ruim e 9-10 para uma condição potencialmente fatal. Assim, cada categoria recebe um escore e a soma total dos escores resulta na classificação da severidade da doença. Os pontos de corte da severidade da doença renal foram: 0 a 10 baixo risco, 11 a 30 médio risco, e acima de 30 alto risco.

Após a coleta, os dados foram armazenados em banco de dados, utilizando o programa Excel versão 2010. Para análise dos dados, foi utilizada estatística descritiva, além de quatro ferramentas estatísticas distintas para verificar a associação entre as variáveis: Quiquadrado (p-valor), V de Cramer, ANOVA (p-valor) e Correlação Intraclasse.

O estudo obedeceu às exigências da Resolução $n^{\circ}$ 466/2012 do CNS e obteve a aprovação do Comitê de Ética em Pesquisa da Fundação de Ensino e Pesquisa das Ciências da Saúde (FEPECS) sob o parecer $n^{\circ}$ : 2.028.465, e Certificado de Apresentação para Apreciação Ética CAAE n ${ }^{\circ}$ 66567517.5.0000.5553.

\section{RESULTADOS}

Dos 54 pacientes analisados, 53,70\% eram mulheres (29) e 44,44\% homens (25). A idade teve média de 52,87 anos, com a faixa etária predominante de 40 a 59 anos (53,70\%). Cerca de 72,22\% (39) declararam-se negros e pardos, houve predominância entre solteiros com 50\% da amostra. Quanto de escolaridade, 55,55\% (30) possuíam o ensino fundamental e 7\% (04) o ensino superior. Mais de 51,87\% (28) eram solteiros. A maioria era beneficiária pelo INSS 74,07\% (40) com renda mensal $\leq 1$ salário 38,88\% (21). Os dados sociodemográficos são apresentados na Tabela 1.

Tabela 1. Variáveis sociodemográficas dos pacientes renais crônicos em HD

\begin{tabular}{|c|c|c|}
\hline VARIA VÉIS & $\mathbf{N}$ & $\%$ \\
\hline \multicolumn{3}{|l|}{ Gênero } \\
\hline Feminino & 29 & $53,70 \%$ \\
\hline Masculino & 25 & $44,44 \%$ \\
\hline \multicolumn{3}{|l|}{ Idade } \\
\hline $20-39$ & 10 & $18,51 \%$ \\
\hline $40-59$ & 29 & $53,70 \%$ \\
\hline$>60$ & 15 & $27,77 \%$ \\
\hline \multicolumn{3}{|l|}{ Etnia } \\
\hline Branca & 14 & $25,92 \%$ \\
\hline Negra & 20 & $37,03 \%$ \\
\hline
\end{tabular}




\begin{tabular}{|c|c|c|}
\hline Parda & 19 & $35,18 \%$ \\
\hline Indígena & 01 & $1,85 \%$ \\
\hline \multicolumn{3}{|l|}{ Escolaridade } \\
\hline Fundamental* & 30 & $55,55 \%$ \\
\hline Médio* & 18 & $33,33 \%$ \\
\hline Superior* & 04 & $7,40 \%$ \\
\hline ND** & 02 & $3,70 \%$ \\
\hline \multicolumn{3}{|l|}{ Estado Civil } \\
\hline Casado & 20 & $37,03 \%$ \\
\hline Solteiro & 28 & $51,85 \%$ \\
\hline Divorciado & 06 & $11,11 \%$ \\
\hline \multicolumn{3}{|l|}{ Ocupação } \\
\hline Segurados pelo INSS & 40 & $74,07 \%$ \\
\hline Ocupação Remunerada & 10 & $18,51 \%$ \\
\hline Desempregado & 01 & $1,85 \%$ \\
\hline Estudante & 01 & $1,85 \%$ \\
\hline ND** & 02 & $3,70 \%$ \\
\hline \multicolumn{3}{|l|}{ Renda } \\
\hline$\leq 1$ salário & 21 & $38,88 \%$ \\
\hline > 1- 2 salários & 16 & $29,62 \%$ \\
\hline > 2-3 salários & 02 & $3,70 \%$ \\
\hline$\geq 3$ salários & 05 & $9,25 \%$ \\
\hline ND & 10 & $18,51 \%$ \\
\hline
\end{tabular}

Referente aos dados clínicos, a nefropatia hipertensiva foi a maior causa de DRC na amostra $(38,88 \%)$, cerca de $44,44 \%$ dos pacientes possuíam cateter temporário para hemodiálise, mais da metade da amostra $(55,55 \%)$ realizava tratamento hemodialítico há menos de 1 ano. Quando questionados se foram submetidos à Dialise Peritoneal (DP) prévia, cerca de $90,74 \%$ relataram que não a realizaram (Tabela 2).

Tabela 2. Perfil clínico dos pacientes renais crônicos em HD

\begin{tabular}{|c|c|c|}
\hline VARIA VÉIS & $\mathbf{N}$ & $(\%)$ \\
\hline \multicolumn{3}{|l|}{ Etiologia da DRC } \\
\hline Nefropatia hipertensiva & 21 & $38,88 \%$ \\
\hline Nefropatia diabética & 12 & $22,22 \%$ \\
\hline Outras causas & 21 & $38,88 \%$ \\
\hline
\end{tabular}




\begin{tabular}{|c|c|c|}
\hline Cateter temporário & 24 & $44,44 \%$ \\
\hline Fístula Arteriovenosa & 22 & $40,74 \%$ \\
\hline Cateter de longa permanência & 07 & $12,96 \%$ \\
\hline Enxerto arteriovenoso & 01 & $1,85 \%$ \\
\hline \multicolumn{3}{|l|}{ Tempo de HD } \\
\hline$\leq 1$ ano & 30 & $55,55 \%$ \\
\hline 1-5 anos & 14 & $25,92 \%$ \\
\hline$\geq 5$ anos & 10 & $18,51 \%$ \\
\hline \multicolumn{3}{|l|}{ Submetido a DP prévia } \\
\hline Sim & 05 & $9,25 \%$ \\
\hline Não & 49 & $90,74 \%$ \\
\hline \multicolumn{3}{|l|}{ Pressão Arterial Sistêmica (PAS) } \\
\hline Normal $(\leq 120)$ & 12 & $22,22 \%$ \\
\hline Pré-hipertensão (121-139) & 7 & $12,96 \%$ \\
\hline HAS estágio 1 (140-159) & 19 & $35,18 \%$ \\
\hline HAS estágio 2 (160-179) & 12 & $22,22 \%$ \\
\hline HAS estágio $3(\geq 180)$ & 04 & $7,40 \%$ \\
\hline \multicolumn{3}{|l|}{ Pressão Arterial Diastólica (PAD) } \\
\hline Normal $(=<80)$ & 31 & $57,40 \%$ \\
\hline Pré-hipertensão (81-89) & 03 & $5,55 \%$ \\
\hline HAS estágio 1 (90-99) & 12 & $22,22 \%$ \\
\hline HAS estágio 2 (100-109) & 04 & $7,40 \%$ \\
\hline HAS estágio 3 (=>110) & 04 & $7,40 \%$ \\
\hline \multicolumn{3}{|l|}{ LDL } \\
\hline Ótimo (<100mg/dL) & 36 & $66,66 \%$ \\
\hline Normal (101-160mg/dL) & 14 & $25,92 \%$ \\
\hline Alto $(161-190 \mathrm{mg} / \mathrm{dL})$ & 02 & $3,70 \%$ \\
\hline Muito alto (>190mg/dL) & 02 & $3,70 \%$ \\
\hline \multicolumn{3}{|l|}{ HDL } \\
\hline Baixo (<40 mg/dL) & 39 & $72,22 \%$ \\
\hline Normal (41 -60 mg/dL) & 10 & $18,51 \%$ \\
\hline Ótimo (> 60 mg/dL) & 05 & $9,25 \%$ \\
\hline \multicolumn{3}{|l|}{ Índice de Massa Corporal (IMC) } \\
\hline Baixo peso $(<18,5)$ & 05 & $9,25 \%$ \\
\hline Peso adequado $(>=18,5-24,9)$ & 33 & $61,11 \%$ \\
\hline Sobrepeso $(>=25-29,9)$ & 13 & $24,07 \%$ \\
\hline Obesidade $(>=30)$ & 03 & $5,55 \%$ \\
\hline
\end{tabular}

Legendas: LDL (Low Density Lipoprotein) /HDL (High Density Lipoprotein): Diretriz Brasileira de Dislipidemias e Prevenção da Aterosclerose (2013) ${ }^{(12)}$

IMC (Índice de Massa Corporal): Diretriz Brasileira Obesidade (2016) ${ }^{(13)}$

PAS (Pressão Arterial sistólica) e PAD (Pressão Arterial Diastólica):7ª Diretriz Brasileira de Hipertensão arterial (2016) ${ }^{(14)}$ 
Nos dados da Tabela 2, observa-se os níveis pressóricos dos pacientes, sendo que 64,8\% possuem a Pressão Arterial Sistólica (PAS) e 37,03\% a Pressão Arterial Diastólica (PAD) não controladas. Quanto ao nível de colesterol fora dos níveis desejáveis, apenas 7,40\% dos pacientes possuem LDL em altos níveis e mais de 72\% possuíam HDL em níveis abaixo do recomendado. Verificou-se que a maioria dos pacientes $(61,11 \%)$ possuíam IMC adequados quando calculado o IMC.

Quanto aos fatores de risco (Tabela 3), verifica-se que a hipertensão arterial (88,88\%), o sedentarismo $(74,07 \%)$ e o consumo elevado de sódio $(74,07 \%)$ foram os fatores de risco cardiovasculares predominantes na população estudada.

Tabela 3. Fatores de risco dos pacientes renais crônicos em HD

\begin{tabular}{|c|c|c|}
\hline FATORES DE RISCO & $\mathbf{N}$ & $(\%)$ \\
\hline Hipertensão Arterial & 48 & $88,88 \%$ \\
\hline Consumo elevado de sódio & 40 & $74,07 \%$ \\
\hline Sedentarismo & 40 & $74,07 \%$ \\
\hline Tabagismo & 24 & $44,44 \%$ \\
\hline Diabetes Mellitus & 22 & $40,74 \%$ \\
\hline $\begin{array}{l}\text { ICC (Insuficiência Cardíaca Congestiva) + } \\
\text { IAM (Infarto Agudo do Miocárdio) }\end{array}$ & 19 & $33,33 \%$ \\
\hline AVC (Acidente Vascular Cerebral) & 08 & $14,81 \%$ \\
\hline
\end{tabular}

Ao analisarmos a classificação do risco cardiovascular segundo o Escore de Risco de Framingham (ERF), nota-se o predomínio entre risco cardiovascular médio e alto $(61,10 \%)$.

Ao compararmos com o Índice de Severidade da Doença Renal (ISDR), grande parte da amostra possui médio e alto risco para severidade da DRC, cerca de 45,59 e 38,88\% respectivamente. (Tabela 4$)$

O ISDR utilizado no estudo, avaliou a severidade da doença renal dos pacientes renais crônicos, apontando que 45,59\% dos renais crônicos possuem alto risco para severidade da DRC. A média geral dos escores foi de risco médio sendo que a diminuição da acuidade visual, e acesso para a diálise foram as que mais contribuíram para compor este índice, seguidas de doença óssea, doença vascular periférica e neuropatia periférica. 
Tabela 4. Escore de Framingham e Índice de Severidade da Doença Renal em pacientes renais crônicos em HD

\begin{tabular}{|l|ccc|}
\hline \multicolumn{1}{|c|}{ ERF } & $\mathbf{N}(\boldsymbol{\%})$ & ISDR & $\mathbf{N}(\%)$ \\
\hline Baixo risco & $21(38,88 \%)$ & Baixo risco & $10(18,51 \%)$ \\
\hline Intermediário Risco & $21(38,88 \%)$ & Médio risco & $21(38,88 \%)$ \\
\hline Alto Risco & $12(22,22 \%)$ & Alto Risco & $23(45,59 \%)$ \\
\hline
\end{tabular}

Quando comparadas as variáveis e o ERF para alto risco cardiovascular, podemos notar que houve associação estatística entre as variáveis consumo elevado de sódio ( $\mathrm{p}=0,02$ e 0,37 ) e ao ISDR ( $\mathrm{p}=0,02$ e 0,32), utilizando o Qui-quadrado e V de Cramer. As demais variáveis não apresentaram associação estatística (tempo de HD, IMC, tempo de HAS, tempo de DM, ICC+IAM, sedentarismo, idade, Pressão Arterial, LDL, HDL, tabagismo).

Pode ser observado na Tabela 5 , que existe uma relação significativa $(p=0,02)$ entre os dois testes utilizados, ERF e ISDR, utilizando a categoria de alto risco cardiovascular do ERF.

Tabela 5: Alto Risco Cardiovascular (ERF) e as variáveis clínicas em pacientes crônicos em HD

\begin{tabular}{|c|c|c|c|c|}
\hline Variável & $\begin{array}{l}\text { Qui- } \\
\text { quadrado } \\
\text { (p-valor) }^{1}\end{array}$ & V de Cramer ${ }^{1}$ & $\begin{array}{l}\text { ANOVA } \\
(p-\text { valor })^{2}\end{array}$ & $\begin{array}{l}\text { Correlação } \\
\text { Intraclasse }^{2}\end{array}$ \\
\hline Tempo de HD & 0,1552 & 0,2630 & & \\
\hline IMC & 0,8869 & 0,1470 & & \\
\hline Tempo de HAS & & & 0,9700 & 0,0000 \\
\hline Tempo de DM & & & 0,7460 & 0,0000 \\
\hline ICC+IAM & 0,3647 & 0,1930 & & \\
\hline Sedentarismo & 0,0761 & 0,3090 & & \\
\hline Consumo elevado de sódio & 0,0249 & 0,3700 & & \\
\hline ISDR (Classificação alto risco) & 0,0223 & 0,3250 & & \\
\hline Idade & & & 0,3550 & 0,0032 \\
\hline PA (Categoria) & 0,7573 & 0,2150 & & \\
\hline $\mathbf{L D L}$ & & & 0,3740 & 0,0002 \\
\hline HDL & & & 0,2330 & 0,0278 \\
\hline Tabagismo & 0,3547 & 0,1960 & & \\
\hline
\end{tabular}




\section{DISCUSSÃO}

Houve predomínio do gênero feminino (53,70\%), em divergência quando comparado a outros estudos realizados com pacientes renais nos quais o gênero masculino é predominante. ${ }^{15-16}$ No Censo Brasileiro de Diálise Crônica de 2016, participaram 309 Unidades de Diálise que contabilizaram uma amostra de 50.807 pacientes, destes 57\% dos pacientes eram do gênero masculino. ${ }^{2} \mathrm{O}$ mesmo ocorreu nos estudos de Cesarino et al, López-Soracipa et al e Cosson et al, nos quais predominou o gênero masculino. Contudo o estudo de DIAS et al, realizado em uma clínica de diálise no MA, apontou que cerca de $63,6 \%$ dos pacientes eram do gênero feminino corroborando com o estudo. ${ }^{8,17-18}$

Em relação à etnia, houve prevalência entre negra e parda (72,22\%), estando em divergência de acordo a literatura em que a DRC tem acometido mais indivíduos brancos. Em um estudo realizado em São Paulo, a prevalência era 60,7\% de indivíduos declarados brancos. ${ }^{8}$ Quando analisada a faixa etária predominante, $o$ mesmo ocorreu no Censo Brasileiro de Diálise Crônica de 2016, em que 65,7\% possuíam idade inferior a 60 anos. $^{2}$ O baixo nível de instrução $(55,55 \%)$ corroborou com outros estudos, assim, como no estudo realizado em Teresina- PI, que 53,13\% possuíam ensino fundamental. ${ }^{16}$

Em relação ao diagnóstico da DRC, a Nefropatia Hipertensiva $(38,88 \%)$ foi a principal etiologia encontrada, seguida da Nefropatia Diabética $(22,22 \%)$, o que corrobora com o Censo Brasileiro de Diálise Crônica de 2016, no qual a hipertensão (34\%) foi a principal causa da doença renal. ${ }^{2}$ O Diabetes mellitus é a segunda maior causa de DRC entre pacientes em HD no Brasil e a mais frequente no mundo. ${ }^{8}$

O percentual de pacientes em HD utilizando cateter de curta permanência seguido de Fístula arteriovenosa (FAV) foram 44,44\% e 40,74\%, respectivamente. Esses achados são maiores quando comparados ao Inquérito Brasileiro de 2016, no qual cerca de 9,4\% utilizavam cateter de curta permanência ${ }^{2}$. Quando comparado o tempo de HD, predominou aqueles que realizavam a terapia a menos de 1 ano, de encontro com um estudo realizado no Maranhão em que a maioria dos pacientes realizavam HD por um período superior a 1 ano, essa divergência pode estar relacionada às características assistenciais do campo de estudo da pesquisa ${ }^{19}$.

O índice de pacientes que desconheciam a DP $(90,74 \%)$ entra em concordância com um estudo realizado com pessoas em tratamento conservador para DRC em que 45\% dos 
renais crônicos afirmaram desconhecer a modalidade ${ }^{20}$. De acordo com o censo da SBN no ano de 2016 , cerca de $92,1 \%$ dos pacientes realizava hemodiálise e $7,9 \%$ diálise peritoneal ${ }^{2}$.

Ao analisarmos os níveis pressóricos, mais da metade dos indivíduos (64,8\%) estão com a PAS não controlada, seguidos de $37,03 \%$ da PAD, significando que mais da metade da amostra do estudo possui risco para desenvolver doenças cardiovasculares. Entre os fatores de risco observados, a hipertensão arterial, o sedentarismo e o consumo elevado de sódio apresentaram alta incidência nos pacientes renais crônicos, cerca de 88,88\%,74,07\% e 74,07\% respectivamente. De acordo com a VII Diretriz Brasileira de Hipertensão Arterial (2016), ocorreram 1.138.670 óbitos em 2013, dos quais $339.672(29,8 \%)$ decorrentes de DCV. A hipertensão não controlada representa um dos maiores fatores de risco para as doenças cardiovasculares ${ }^{14}$. No estudo de Cesarino et al, a hipertensão arterial, o sedentarismo e o tabagismo foram os fatores de risco predominantes, corroborando com os dados encontrados, no presente estudo ${ }^{8}$.

Segundo recomendações da Sociedade Brasileira de Nefrologia (SBN) como estratégias para controle de tal fatores de risco, deve-se instigar os pacientes quanto a prática de atividade física regular, manter uma dieta equilibrada com menor teor de sódio e gorduras, uso contínuo e de acordo a prescrição médica dos anti-hipertensivos, cessação do tabagismo e consumo de álcool, para assim promover a redução da morbidade e mortalidade das doenças cardiovasculares.

Contudo, a má aderência ao tratamento representa um obstáculo e resulta em falha terapêutica. ${ }^{8,14}$. Para realizar esta abordagem, estudos comprovaram que a atuação da equipe multiprofissional promove melhor controle da HA, o que está diretamente relacionado à adesão ao tratamento medicamentoso e não medicamentoso, associando medidas para o sedentarismo e $\operatorname{dieta}^{8}$.

Níveis elevados de LDL e baixos de HDL são fatores agravantes para doenças cardiovasculares, pois o LDL contribui para formação de placas ateroscleróticas e o HDL em níveis baixos deixa de exercer função protetora contra a aterogênese ${ }^{12}$.Neste contexto, observa-se que os indivíduos da amostra possuem baixos níveis de LDL e altos níveis para HDL, o que não oferece assim risco cardiovascular quanto a esses critérios ${ }^{16}$. Outro fator no qual representa risco cardiovascular é a obesidade, relacionada ao risco coronariano e no desenvolvimento da hipertrofia ventricular esquerda ${ }^{21}$. No presente estudo, prevaleceu o IMC 
adequado entre os renais crônicos dialíticos corroborando com os dados encontrados em um estudo realizado com renais crônicos em que $41,3 \%$ possuíam peso normal ${ }^{8}$.

Ao considerarmos a classificação do risco cardiovascular segundo o ERF, predominou o médio e alto risco cardiovascular $(61,10 \%)$ o que está em discordância com um estudo realizado com renais crônicos em HD, em que em $62 \%$ dos pacientes predominavam o baixo risco cardiovascular ${ }^{8}$.

O Instrumento utilizado para avaliação do risco cardiovascular foi o ERF tradicional, embora ainda exista o ERF modificado, que incorpora fatores como a presença de hipertrofia de ventrículo esquerdo, níveis de microalbuminúria e creatina, além daqueles utilizados no ERF tradicional. A escolha do instrumento tradicional se deve à disponibilidade dos recursos dispostos no local da coleta de dados, no qual não seriam possíveis os resultados dos fatores: hipertrofia de ventrículo esquerdo e os níveis de microalbuminúria desses pacientes, utilizados no ERF modificado ${ }^{8,22,23}$.

A presença de comorbidades em pacientes renais crônicos em HD é reconhecida como determinante de sobrevida e morbidade desses pacientes. Ao analisar a severidade da doença renal utilizando o ISDR, foi apontado que 45,59\% e 38,88\% dos indivíduos do estudo possuem médio e alto risco para severidade da DRC respectivamente. As variáveis que mais contribuíram para compor este índice foram: diminuição visual e acesso para diálise, seguidas de doença óssea, doença vascular periférica, neuropatia periférica e DM.

Craven et al, ao estudar as comorbidades dos pacientes em HD utilizando o ISDR, observou que os pacientes que foram a óbito em seis meses de tratamento possuíam escores médios de 18 pontos, enquanto os sobreviventes possuíam escores médio de $9^{(11)}$. Cerca de $45,59 \%$ dos pacientes do estudo apresentaram escores superiores a 31, caracterizando uma população de alta morbidade.

No estudo de Morsch C. et al, 33\% dos pacientes em HD apresentaram escores superiores a 31, caracterizando uma população de alta morbidade, em concordância com nosso estudo. ${ }^{3}$ Esse escore também foi maior nos pacientes diabéticos, no qual 50\% dos pacientes diabéticos do estudo possuíam escore maiores de 31. Os pacientes renais crônicos diabéticos tiveram escores maiores do ISDR, que pode estar relacionado às inúmeras comorbidades que estes pacientes apresentam, especialmente cardiovasculares. 
Houve associação estatística entre ERF e o ISDR $(\mathrm{p}=0,02)$, contudo sem grau significativo $(0,32)$, pois apesar dos dois instrumentos utilizarem categorias semelhantes (doença cardíaca e DM), avaliam as variáveis de formas diferentes. Enquanto o ERF avalia os níveis pressóricos e a presença de DM, o ISDR avalia o grau de comorbidade cardíaca verificando sinais e sintomas como angina e ICC, a utilização de hipoglicemiantes e grau de severidade da hipoglicemia dos pacientes.

Quando comparados o risco cardiovascular e a severidade da doença renal dos pacientes renais crônicos em HD, de acordo com o ERF, prevaleceu o baixo e médio risco cardiovascular (ambos 38,88\% dos pacientes do estudo). As variáveis: LDL, idade, PAD, DM e tabagismo atingiram o percentual que se enquadram na categoria de baixo risco $(<10 \%$ do risco) e médio risco (10 a 20\% do risco). Segundo o ISDR, predominou o alto risco (45,59\% dos pacientes do estudo) devido as variáveis: diminuição visual, neuropatia autonômica e acesso vascular, que foram analisadas por apresentarem pontos que satisfazem a classificação de alto risco do ISDR (>30 pontos do escore) e não serem utilizadas no cálculo do ERF.

Em um estudo realizado na Bahia, as variáveis sedentarismo x risco cardiovascular e, tempo de hemodiálise x risco cardiovascular obtiveram valor estatisticamente significativo, em discordância com nosso estudo, que não apresentou relação estatística significativa (p= 0,076 e $\mathrm{p}=0,155$ ) para sedentarismo e tempo de hemodiálise, o que pode estar relacionado ao tamanho da amostra do estudo ${ }^{22}$

\section{CONCLUSÃO}

O estudo analisou o risco cardiovascular e a severidade da doença renal dos pacientes crônicos submetidos à HD utilizando o ERF e a ISDR.

Foi evidenciado que $61,10 \%$ da amostra do estudo apresentou médio e alto risco cardiovascular, sendo que os fatores de risco sedentarismo, consumo elevado de sódio e hipertensão arterial apresentaram os maiores índices no escore. De acordo com o ISDR, $84,47 \%$ da amostra apresentaram médio e alto risco para severidade da DRC, sendo que as variáveis com maiores pontuações foram aquelas inerentes ao tratamento dialítico: diminuição visual, acesso para diálise e neuropatia autonômica. 
Houve associação estatística entre o ERF e as variáveis consumo elevado de sódio e ao ISDR e não houve associação estatística com as demais variáveis. Há poucos estudos na literatura abordando o tema, sendo necessárias mais pesquisas nesta área. Não foram encontrados estudos relacionando o ERF com as variáveis tempo de HAS, ICC, IAM e IMC.

O índice elevado de mortalidade por doenças cardiovasculares em renais crônicos submetidos à HD, traz à reflexão, a avaliação do risco cardiovascular na prática clínica no âmbito dessa terapia. A avaliação deste risco deve ser segura, regular e dinâmica, observando fatores de risco presentes. A avaliação da severidade da doença renal também é necessária, pois a mortalidade da doença renal está relacionada com a gravidade da doença e ao grau de distúrbios fisiológicos. Dessa forma, instrumentos para avaliar a severidade são necessários para individualizar cuidados a esses pacientes.

Entre os diversos métodos de avaliação cardíaca, sugere-se o ERF que, apesar de possuir limitações, é um instrumento valioso e contribui para tomada de decisões da equipe, também sendo indicado utilizar o ERF modificado, que incorpora outros fatores importantes para o diagnóstico do risco cardiovascular.

Existem poucos instrumentos que avaliam a gravidade e comorbidades da doença renal e poucos foram utilizados em pacientes com doença renal crônica. O ISDR é um instrumento que avalia a severidade da doença renal que contribui para identificar a gravidade da doença, diferenciar a severidade por paciente, realizar um planejamento terapêutico e avaliação da melhora ou piora do quadro da doença renal.

Dentre as limitações do estudo, elenca-se a falta de alguns exames laboratoriais disponíveis na unidade, imprescindíveis para a realização da pesquisa e um fator restritivo para o estudo, e a impossibilidade de aplicação do questionário a um número maior de pacientes.

O Escore de Framingham e o ISDR mostraram-se ferramentas producentes na avaliação do risco cardiovascular e da severidade da doença renal. Sugere-se o uso em outras pesquisas, assim como a associação dos dois métodos.

Estudos dessa temática evidenciam a situação de risco cardiovascular que pacientes renais crônicos dialíticos se encontram, de forma a instigar a equipe a promover ações de prevenção e tratamento. Contribuem também como forma de intervenção preventiva de DCV 
à essa população e acrescentam dados estatísticos da situação local de uma unidade de terapia hemodialítica para estudos futuros.

Diante da situação de risco cardiovascular e da severidade da doença renal, deve-se priorizar a participação da equipe multidisciplinar a fim de buscar minimizar riscos e promover o acompanhamento da função cardiovascular dos renais crônicos em HD haja vista a necessidade de levantamento de estratégias para promover a adesão ao tratamento no que tange as ações medicamentosas e não medicamentosas para melhorar a sobrevida e a qualidade de vida dessa população.

\section{REFERÊNCIAS}

1. Ministério da Saúde. Secretaria de Atenção à Saúde. Departamento de Atenção Especializada e Temática. Diretrizes Clínicas para o Cuidado ao paciente com Doença Renal Crônica - DRC no Sistema Único de Saúde. Brasília (Brasil): Ministério da Saúde, 2014. $37 \mathrm{p}$.

2. Sesso RC, et al. Inquérito Brasileiro de Diálise Crônica 2016. J Bras Nefrol. 2017; 39(3):261-66. Disponível em: http://www.scielo.br/pdf/jbn/v39n3/pt_0101-2800-jbn39-03-0261.pdf

3. Morsch $\mathrm{C}$, et al. Índice de gravidade da doença renal, indicadores assistenciais e mortalidade em pacientes em hemodiálise. Rev. Assoc. Med. Bras. Porto Alegre, RS. 2005 Outubro; 51(5). Available from: http://dx.doi.org/10.1590/S010442302005000500023

4. Riella MC. Princípios de Nefrologia e Distúrbios hidroeletrolíticos. $5^{\circ}$ ed. Rio de Janeiro: Guanabara, 2014.

5. Franco LC. A Doença Renal como fator de Risco Cardiovascular. Arquivos Brasileiros de Cardiologia. 2005; 85(6):432-436. Disponível em: http://www.scielo.br/scielo.php?script=sci_arttext\&pid=S0066-782X2005001900011 
6. Winter D, et al. Sobrevida e fatores de risco de mortalidade em pacientes sob hemodiálise. HU Revista. Juiz de Fora. 2016 Dezembro;42(4): 267-275. Disponível em: https://hurevista.ufjf.emnuvens.com.br/hurevista/article/viewFile/2483/902

7. I Diretriz Brasileira de Prevenção Cardiovascular. Arquivos Brasileiros de Cardiologia. 2013 Dezembro; 101(6). Disponível em: http://www.scielo.br/scielo.php?script=sci_arttext\&pid=S0066-782X2013004500001

8. Cesarino $\mathrm{CB}$ et al. Avaliação do risco cardiovascular de pacientes renais crônicos segundo critérios de Framingham. Acta Paul Enferm, São José do Rio Preto, SP. 2013; 26(1): 101-107. Disponível em: www.scielo.br/scielo.php?script=sci_arttext\&pid=S0103-21002013000100016

9. Passos PRC, Motta EAP, Gonçalves GOL. Avaliação segundo o índice de risco de Framingham de pacientes hemodialíticos em terapia com calcitriol. Rev. Ciênc. Saúde, São Luís. 2014 jul-dez;16(2): 66-72. Disponível em: http://www.periodicoseletronicos.ufma.br/index.php/rcisaude/article/view/4059

10. Mafra F, Oliveira H. Risk Assessment cardiovascular - methodologies and its implications on clinical practice. Rev. Port. Clin. Geral.2008; 24:391-400. Disponível em: http://www.rpmgf.pt/ojs/index.php/rpmgf/article/view/10509

11. Craven J. et al. The end-stage renal disease severity index (ESRD_SI). Psycological Medicine. $1991 ;$ 21: 237-243. Disponível em: https://www.ncbi.nlm.nih.gov/pubmed/2047501

12. Xavier HT, et al. V Diretriz Brasileira de Dislipidemias e Prevenção da Aterosclerose. Arq. Bras. Cardiologia. SP. 2013 outubro; 101(4). Disponível em: http://www.scielo.br/scielo.php?pid=S0066782X2013004100001\&script=sci_arttext\#e nd 
13. Diretriz Brasileira de Obesidade. Associação Brasileira para o estudo da obesidade e da síndrome metabólica. São Paulo. 2016.

14. Sociedade Brasileira de Cardiologia. VII Diretriz Brasileira de Hipertensão Arterial. 2016 setembro; 107(3). Disponível em: http://publicacoes.cardiol.br/2014/diretrizes/2016/05_HIPERTENSAO_ARTERIAL.p df

15. Mendonça $\mathrm{AE}$, et al. Perfil sociodemográfico e clínico de idosos submetidos à hemodiálise. Cogitare Enferm. Natal, RN. 2015 Jan/Mar; 20(1):60-6. Disponível em: http://revistas.ufpr.br/cogitare/article/view/37080

16. Ribeiro IP, et al. Perfil Epidemiológico dos portadores de insuficiência renal crônica submetidos à terapia hemodialítica. Enferm. Foco. 2014; 5(3/4): 65-69. Disponível em: http://revista.portalcofen.gov.br/index.php/enfermagem/article/view/567/249

17. Dias EC, et al. Avaliação dos índices de infecção relacionados ao cateter duplo lúmen para hemodiálise antes e após orientação para o autocuidado. Revista UNINGÁ. Teresina, PI. 2017 Jul/Set; 53(2): 18-25. Available from: https://www.mastereditora.com.br/download-2415

18. Cosson IO, et al. Perfil dos pacientes em terapia renal substitutiva em unidade de nefrologia. Rev enferm UFPE on line. Recife. 2014 outubro; 8(2):3693-9. Disponível em:https://periodicos.ufpe.br/revistas/revistaenfermagem/article/download/10111/1058 6

19. Ribeiro PRS, Batista TS. Adesão ao tratamento farmacológico anti-hipertensivo de pacientes em hemodiálise. Rev. Ciências Farm. Básica Apl. Imperatriz, MA. 2015; 36(2): 201-2012. Disponível em: http://seer.fcfar.unesp.br/rcfba/index.php/rcfba/article/view/233 
20. Santos RLG, et al. Avaliação do conhecimento do paciente renal crônico em tratamento conservador sobre modalidades dialíticas. Rev. Enferm. UFPE on line. Recife. 2015 fevereiro; $9(2): \quad 652-660 . \quad$ Disponível em: https://periodicos.ufpe.br/revistas/revistaenfermagem/article/viewFile/10384/11134

21. Carvalho CA, et al. Associação entre fatores de risco cardiovascular e indicadores antropométricos de obesidade em universitários de São Luís, Maranhão. Ciências \& Saúde Coletiva. 2015; 20(2):479-490. Disponível em: www.scielo.br/pdf/csc/v20n2/1413-8123-csc-20-02-0479.pdf

22. Caracas DRS, et al. Prevalência de risco cardiovascular e nível de atividade física em pacientes com doença renal crônica em tratamento hemodialítico. Rev. Saúde.Com. Bahia. 2017; 13(2): 871-878. Disponível em: www.uesb.br/revista/rsc/ojs/index.php/rsc/article/view/420/465

23. Jürge F, et al. Development and validation of a predictive mortality risk score from a European hemodialysis cohort. Kidney Int. 2015 may; 87(5): 996-1008. Disponível em: http://www.kidneyinternational-online.org/article/S0085-2538(15)30105-8/abstract 